Rodolpho Cruz VieIRA ${ }^{1}$

IONARA Diniz Evangelista

Santos Barcelos ${ }^{1}$

Elisa Melo Ferreira

Maria Cristina Picinato

Medeiros de Araújo

Rosana Maria dos Reis ${ }^{3}$

RUI AlberTo FerRIAN ${ }^{4}$

Paula Andrea de Albuquerque

SAlles NAVARro ${ }^{5}$

Artigos originais

Palavras-chave

Síndrome do ovário policístico

Oócitos/análise

Injeções de esperma intracitoplásmicas Indução de ovulação Infertilidade feminina Infertilidade masculina

Keywords

Polycystic ovary syndrome Oocytes/analysis Sperm injections, intracytoplasmic

Ovulation induction Infertility, female Infertility, male

Correspondência:

Paula Andrea de Abuquerque Salles Navarro Setor de Reprodução Humana, Departamento de Ginecologia e Obstetrícia da Faculdade de Medicina de Ribeirão Preto da Universidade de São Paulo Avenida Bandeirantes, 3.900 - Monte Alegre (EP 14049-900 - Ribeirão Preto (SP), Brasil. Fone: (16) 3602-2821/Fax: (16) 3602-2810 E-mail: pnavarro@fmrp.usp.br

Recebido

$14 / 2 / 08$

Aceito com modificacōes

15/5/08

\section{Avaliação de anomalias meióticas de oócitos em pacientes com síndrome dos ovários policísticos submetidas à estimulação ovariana}

\author{
Evaluation of meiotic abnormalities of oocytes from polycystic ovary \\ syndrome patients submitted to ovarian stimulation
}

\section{Resumo}

OBJETIVO: avaliar o fuso meiótico e a distribuição cromossômica de oócitos maturados in vitro, obtidos de ciclos estimulados de mulheres inférteis com síndrome dos ovários policísticos (SOP) e fatores masculino e/ou tubário de infertilidade (Grupo Controle) e comparar as taxas de maturação in vitro (MIV) entre os dois grupos avaliados. MÉTODOS: cinco pacientes inférteis com SOP e oito pacientes controles, submetidas à estimulação ovariana para injeção intracitoplasmática de espermatozóide, foram selecionadas prospectiva e consecutivamente, e constituíram os grupos de estudo e Controle, respectivamente. Oócitos imaturos captados após estimulação ovariana para a realização de injeção intracitoplasmática de espermatozóide 121 e 29, respectivamente, nos Grupos SOP e Controle) foram submetidos à MIV. Apenas os oócitos que apresentaram a extrusão do primeiro corpúsculo polar após a MIV foram fixados e submetidos à imunocoloração e análise por microscopia de fluorescência para avaliação morfológica do fuso e da distribuição cromossômica. A análise estatística foi realizada utilizando o teste exato de Fisher, com significância estatística quando $\mathrm{p} \leq 0,05$. RESULTADOS: as taxas de MIV foram similares entre os dois grupos (47,6 e 44,8\%, respectivamente, nos Grupos SOP e Controle). Seis dos dez oócitos (60\%) analisados do grupo de estudo e quatro dos 12 oócitos $(33,3 \%)$ analisados do Grupo Controle apresentaram anomalias meióticas, caracterizadas por anomalias do fuso e/ou distribuição cromossômica oocitária, sem diferença significativa entre os grupos. CONCLUSÕES: os dados do presente estudo não demonstraram diferença significativa nas taxas de MIV e nas proporções de anomalias meióticas entre os oócitos maturados in vitro, provenientes de ciclos estimulados de pacientes com SOP, quando comparados aos controles.

\section{Abstract}

PURPOSE: to evaluate the meiotic spindle and the chromosome distribution of in vitro matured oocytes obtained from stimulated cycles of infertile women with polycystic ovary syndrome (PCOS) and with male factor and/or tubal infertility (Control Group) and compare in vitro maturation (IVM) rates between the groups analyzed. METHODS: five infertile patients with PCOS and eight controls, submitted to stimulated cycles for intracytoplasmic sperm injection, were selected prospectively and consecutively, and respectively assigned to the study group and the Control Group. Immature oocytes (21 and 29, respectively, from PCOS and Control Group) were submitted to IVM. After IVM, oocytes with first polar body extruded were fixed and submitted to immunostaining and fluorescence microscopy for morphological evaluation of the spindle and of chromosome distribution. Statistical analysis was performed by the Fisher test with significance, when $\mathrm{p} \leq 0.05$. RESULTS: IVM rates were similar between groups 147.6 e $44.8 \%$, respectively, for PCOS and Control Group). Six of the ten oocytes (60\%) from the study group and four of the 12 oocytes (33.3\%) from the Control Group presented meiotic anomalies of the spindle and/or anomalous chromosome distribution, without statistical difference between groups. CONCLUSIONS: data from the present study did not demonstrate significant difference neither in IVM rates nor in the proportions of meiotic anomalies between in vitro matured oocytes obtained from stimulated cycles from PCOS patients and control ones.
Laboratório de Ginecologia do Hospital das Clínicas da Faculdade de Medicina de Ribeirão Preto, Setor de Reprodução Humana, Departamento de Ginecologia e Obstetrícia da Faculdade de Medicina de Ribeirão Preto da Universidade de São Paulo - USP Ribeirão Preto (SP), Brasil.

' Pós-graduando do Departamento de Ginecologia e Obstetrícia da Faculdade de Medicina de Ribeirão Preto da Universidade de São Paulo - USP - Ribeirão Preto (SP), Brasil.

2 Bióloga do Laboratório de Reprodução Assistida do Departamento de Ginecologia e Obstetrícia da Faculdade de Medicina de Ribeirão Preto da Universidade de São Paulo - USP - Ribeirão Preto (SP), Brasil.

3 Professora Associada do Departamento de Ginecologia e Obstetrícia da Faculdade de Medicina de Ribeirão Preto da Universidade de São Paulo - USP - Ribeirão Preto (SP), Brasil.

${ }_{4}^{4}$ Professor Titular do Departamento de Ginecologia e Obstetrícia da Faculdade de Medicina de Ribeirão Preto da Universidade de São Paulo - USP - Ribeirão Preto (SP), Brasil.

5 Doutora, Professora do Departamento de Ginecologia e Obstetrícia da Faculdade de Medicina de Ribeirão Preto da Universidade de São Paulo - USP - Ribeirão Preto (SP), Brasil. 


\section{Introdução}

Apesar do uso cada vez mais freqüente das técnicas de reprodução assistida (TRA) para o tratamento da infertilidade relacionada à síndrome dos ovários policísticos (SOP), permanece incerto se o sucesso destes procedimentos difere entre as pacientes com e sem esta síndrome. Alguns autores relatam menores taxas de fertilização e maior incidência de abortamentos após a realização de TRA neste grupo de pacientes ${ }^{1,2}$. Questiona-se o papel do comprometimento da receptividade endometrial e da qualidade oocitária na gênese dos efeitos deletérios da SOP sobre a fertilidade feminina ${ }^{3,4}$. Postula-se que as concentrações elevadas de hormônio luteinizante (LH) na fase folicular, ao interferir na foliculogênese, poderiam resultar em oócitos de má qualidades. É possível que outras alterações endócrinas e metabólicas associadas a esta síndrome também possam estar relacionadas com o comprometimento da qualidade oocitária, o que precisa ser elucidado. O comprometimento da qualidade oocitária, por sua vez, poderia contribuir para a redução das taxas de fertilização, observadas após a realização de TRA.

Os estudos que avaliaram de forma não invasiva a qualidade oocitária em pacientes com SOP utilizaram a análise de parâmetros como a taxa de oócitos em metáfase II ${ }^{6-8}$ e a análise da morfologia do complexo cumulus-oócito ${ }^{9,10}$. Contudo, pela fraca correlação destes parâmetros com o desenvolvimento embrionário subseqüente, e, conseqüentemente, com o prognóstico da gestação, supõe-se que estes critérios não sejam os preditores adequados da real qualidade oocitária. Entretanto, não se dispuseram de estudos que tenham avaliado a qualidade oocitária em portadoras de SOP por meio da análise de critérios morfológicos mais objetivos e, potencialmente, capazes de predizer a real capacidade desenvolvimental oocitária.

A qualidade oocitária é decorrente de fatores correlacionados às competências nuclear e citoplasmática. A competência citoplasmática é responsável pela capacidade do oócito de suportar a fertilização e o desenvolvimento embrionário inicial. A competência nuclear é caracterizada pela qualidade da cromatina e do fuso meiótico oocitário, estrutura temporária e dinâmica, constituída por microtúbulos polimerizados, importante na segregação cromossômica e na divisão celular ${ }^{11-13}$. Qualquer alteração no complemento cromossômico, que possa ser originada por uma alteração do fuso meiótico, poderia conduzir a um estado de aneuploidia por não-disjunção, junção desbalanceada ou disjunção prematura das cromátides e perda de cromossomos, comprometendo a fertilização e o subseqüente desenvolvimento embrionário ${ }^{14,15}$. Desta forma, para que o oócito maduro esteja preparado para a fertilização, é necessário que o fuso meiótico, estrutura fundamental no processo de maturação e ativação oocitárias, mantenha sua integridade e funcionalidade. Até o presente momento, não foram dispostos estudos que tenham avaliado a morfologia do fuso celular e distribuição cromossômica oocitária de pacientes com SOP, comparadas a mulheres inférteis sem esta síndrome. Ressalta-se que a avaliação da qualidade oocitária por meio do estudo morfológico da organização e distribuição estrutural do fuso meiótico, responsável pela organização do conteúdo nuclear e também pela divisão celular oocitária, é um método simples, com boa reprodutibilidade e que ajudaria a esclarecer uma das potenciais variáveis relacionadas à piora da qualidade oocitária em portadoras de SOP.

Assim, no presente estudo, avaliaram-se o fuso meiótico e a distribuição cromossômica de oócitos maturados in vitro obtidos de ciclos estimulados de mulheres inférteis com SOP, comparando com os dados obtidos de oócitos de um Grupo Controle, constituído por mulheres com fatores masculino e/ou tubário de infertilidade. Como objetivo secundário, compararamse as taxas de maturação oocitária in vitro, nos Grupos SOP e Controle, de oócitos imaturos, obtidos em ciclos estimulados com gonadotrofinas.

\section{Métodos}

\section{Pacientes e escolha da amostra}

Foram incluídos, no presente estudo, consecutivamente, todos os casais submetidos à estimulação ovariana para a realização de injeção intracitoplasmática de espermatozóides (ICSI), junto ao Setor de Reprodução Humana do Departamento de Ginecologia e Obstetrícia da Faculdade de Medicina de Ribeirão Preto da Universidade de São Paulo (FMRP-USP), no período de abril de 2006 a abril de 2007, que preencheram os critérios de inclusão abaixo descritos e manifestaram o desejo de participar do projeto, mediante a assinatura do termo de consentimento pós-informado.

Foram incluídas pacientes inférteis com idade $\leq 38$ anos, índice de massa corpórea (IMC) $<30 \mathrm{~kg} / \mathrm{m}^{2}$ e FSH basal $\leq 10 \mathrm{mUI} / \mathrm{mL}$, distribuídas em dois grupos: Grupo SOP (síndrome dos ovários policísticos, diagnosticada segundo os critérios definidos pelo Consenso de Rotterdam $)^{16}$, constituído de cinco pacientes com infertilidade secundária à presença exclusiva de anovulação crônica, e Grupo Controle, composto por oito pacientes com infertilidade secundária a fator masculino e/ou tubário (excluídos os casos de hidrossalpinge). Foi considerada como critério de inclusão para ambos os grupos a presença de oócitos imaturos captados e doados que, após maturação in vitro (MIV), tornaram-se maduros (atingiram a metáfase da segunda divisão meiótica, morfologicamente identificada pela presença da extrusão do primeiro corpúsculo polar). 
Durante o período de inclusão, foram obtidos, respectivamente, nos Grupos SOP (14 ciclos estimulados de 12 pacientes) e Controle (30 ciclos estimulados de 23 pacientes), 21 e 29 oócitos imaturos. Após MIV, dez oócitos no Grupo SOP (provenientes de cinco pacientes) e 12 (oriundos de oito pacientes) no Grupo Controle apresentaram a extrusão do primeiro corpúsculo polar, sendo fixados, processados e analisados no presente estudo.

Foram excluídas do estudo pacientes que utilizaram medicamentos que pudessem interferir na foliculogênese ovariana nos dois meses que antecederam o início da estimulação ovariana (como antiinflamatórios não esteroidais e corticosteróides).

A estimulação ovariana seguiu o protocolo do setor, que, sucintamente, inclui a programação do início do ciclo mediante o uso de contraceptivos orais combinados, dessensibilização hipofisária com agonistas do hormônio liberador de gonadotrofinas ( $\mathrm{GnRH}$, protocolo longo), estimulação ovariana controlada com gonadotrofina (FSH recombinante) e administração de gonadotrofina coriônica humana (hCG) 34 a 36 horas antes da realização da captação oocitária.

As pacientes foram submetidas a uma injeção subcutânea diária de 0,5 mg de acetato de leuprolide (Lupron ${ }^{\circledR}$, Abott, Brasil), iniciada dez dias antes da ultra-sonografia basal, previamente ao início da estimulação ovariana. Foram utilizadas diariamente 200 a 300 unidades de FSH recombinante (Gonal-F ${ }^{\circledR}$, Serono, Brasil; Puregon ${ }^{\circledR}$, Organon, Brasil), nos primeiros seis dias da estimulação ovariana. No sétimo dia da estimulação ovariana foi iniciada a monitorização ultra-sonográfica do ciclo. A partir deste momento, o controle ultra-sonográfico foi realizado diariamente ou a cada dois dias, e a dose de gonadotrofinas foi ajustada de acordo com o crescimento folicular observado. A suspensão das gonadotrofinas e do agonista do $\mathrm{GnRH}$ foi realizada quando pelo menos dois folículos apresentavam diâmetro médio de $18 \mathrm{~mm}$, quando foi administrada $250 \mu \mathrm{g}$ de hCG recombinante (Ovidrel $^{\circledR}$, Serono, Brasil) às $22 \mathrm{~h}$. Cerca de 34 a 36 horas após a administração do hCG, a paciente foi submetida à captação oocitária, sob sedação endovenosa com propofol (Diprivan ${ }^{\circledR}$, Astra-Zeneca, Brasil) e citrato de fentanil (Fentanil ${ }^{\circledast}$, Janssen-Cilag, Brasil). A aspiração folicular foi realizada por meio de ultra-sonografia, com transdutor transvaginal de $5 \mathrm{MHz}$ acoplado à guia de punção, sob a forma de pool. O material aspirado foi analisado para a identificação e o isolamento dos complexos oócito-cumulus (COC). Após lavagem cuidadosa, os COC identificados foram colocados em placas NUNC (Multidish 4 wells Nuclon, Delta SI), preenchidas com o meio de cultura Human Tubal Fluid-HEPES (HTF, Irvine Scientific) suplementado com $10 \%$ soro sintético substituto (SSS), cobertas com óleo mineral
(Sigma-Aldrich), e levados à incubadora, em mistura gasosa de $\mathrm{CO}_{2}$ a $5 \%$, sob temperatura de $37^{\circ} \mathrm{C}$ e $95 \%$ de umidade, por um período de duas a quatro horas. Após este período, foi realizado o desnudamento oocitário, utilizando-se a exposição dos COC a hialuronidase (H4272 tipo IV-S, Sigma; $80 \mathrm{UI} / \mathrm{mL}$ ) por 30 segundos, seguida pela remoção mecânica das células do cumulus em meio HTF-SSS, com o auxílio de uma pipeta Stripper (Denuding Pipette, Cook; $130 \mu \mathrm{m}$ ).

Imediatamente após o desnudamento oocitário, realizou-se a análise morfológica para identificação do grau de maturidade dos oócitos, sob visualização ao microscópio de luz. Os oócitos que atingiram a metáfase II (MII, caracterizada morfologicamente pela extrusão do primeiro corpúsculo polar, CP) foram considerados maduros e incubados em gotas de $25 \mu \mathrm{L}$ de HTF + SSS $10 \%$ por uma hora para, então, serem inseminados por meio da realização da ICSI, não sendo, portanto, analisados no presente estudo. Os oócitos que apresentaram sinais de danos mecânicos ou sinais de atresia (citoplasma irregular, escuro ou granulado) foram descartados. Os oócitos que estavam em estágio de vesícula germinativa (VG, ausência de CP extruso e presença de VG) ou de metáfase I (ausência do CP e da VG) foram considerados imaturos, e, portanto, sem utilidade clínica para a realização de ICSI, sendo submetidos à MIV, como descrito a seguir, para utilização no presente estudo.

Para MIV, os oócitos imaturos foram transferidos para placas de cultivo em microgotas de $25 \mu \mathrm{L}$ do meio $\mathrm{HTF}+$ SSS $10 \%$ (meio previamente equilibrado por período $\geq 4$ horas) e levados à incubadora em mistura gasosa de $\mathrm{CO}_{2}$ a $5 \%$, sob condições ideais de temperatura $\left(37^{\circ} \mathrm{C}\right)$ e umidade $(95 \%)$, por período previamente definido. Os oócitos no estágio de vesícula germinativa foram mantidos nestas condições de cultivo por um período de $19 \pm 1$ hora e aqueles em metáfase I, por $4 \pm 0,5$ hora, conforme curva de maturação previamente realizada neste serviço, utilizando os meios e condições de cultura previamente descritos. Após este período pré-estabelecido de cultivo, foi realizada novamente a análise do grau de maturação oocitária. Os oócitos que não atingiram a MII após o período descrito, assim como os que degeneraram, foram descartados. Os oócitos que atingiram o estágio de MII foram fixados para análise por microscopia de imunofluorescência para caracterização do fuso celular e distribuição cromossômica, conforme previamente descrito ${ }^{17,18}$. A taxa de MIV (definida dividindo-se o número de oócitos que atingem a MII pelo número total de oócitos imaturos submetidos à MIV) foi calculada para os dois grupos estudados.

Para a fixação e coloração dos oócitos para a microscopia de imunofluorescência, utilizaram-se reagentes 
adquiridos da Sigma (Sigma Chemical Co., St. Louis, MO), com exceção dos descritos individualmente a seguir. Os oócitos maturados in vitro foram colocados em um fixador adequado para propiciar a estabilidade do fuso celular, composto por um tampão estabilizador (0,1 M Pipes, $5 \mathrm{mM}$ cloreto de magnésio hexahidratado e 2,5 mM etileno glicol tetra-acético ácido, EGTA, 50\% de óxido de deutério, 0,01\% de apronitina de pulmão bovino, $1 \mathrm{mM}$ DTT, $1 \mu \mathrm{M}$ taxol, $0,5 \%$ Triton-X, $2 \%$ formaldeído e água de MilliQ), previamente aquecido a $37^{\circ} \mathrm{C}$ por 30 minutos. Os oócitos foram fixados a $37^{\circ} \mathrm{C}$ por 30 minutos e lavados quatro vezes em solução tampão de lavagem (15 minutos cada) composta por $0,02 \% \mathrm{NaN}_{3}$, $0,01 \%$ Triton-X, $0,2 \%$ leite desnatado, $2 \%$ soro normal de cabra, 0,1 M de glicina, 2\% BSA(V) e PBS, na qual ficaram por duas horas a $37^{\circ} \mathrm{C}$. Após esse período, os oócitos foram lavados uma vez na solução tampão de lavagem para incubação com o anticorpo primário (beta tubulina de camundongo, diluição 1:1.000 em solução de PBS com $0,02 \% \mathrm{NaN}_{3}$ e $\left.0,1 \% \mathrm{BSA}(\mathrm{V})\right)$ por quatro horas a $37^{\circ} \mathrm{C}$. Em seguida, foram lavados mais três vezes (15 minutos cada) e incubados com o anticorpo secundário (imunoglobulina de cabra anti-camundongo FITC-conjugada, Zymed Laboratories, Invitrogen Corporation, Calsbad, CA, USA, diluição 1:200 na mesma solução de diluição acima descrita) por duas horas a $37^{\circ} \mathrm{C}$. Após mais três lavagens de 15 minutos cada, os oócitos foram corados com Hoechst 33342 em meio suporte por dez minutos à temperatura ambiente, montados entre lâmina e lamínula, estocados a $4^{\circ} \mathrm{C}$ e mantidos no escuro até observação em microscópio de imunofluorescência (Zeiss Axiovert 100TV), realizada dentro de uma semana do preparo da lâmina, para análise morfológica do fuso meiótico e da distribuição cromossômica.

O fuso meiótico foi considerado normal quando apresentou a forma de barril e estava disposto em posição centrípeta em relação ao corpúsculo polar. Os cromossomos apresentaram distribuição normal quando estavam reunidos na placa metafásica e bem alinhados no equador do fuso celular oocitário. Consideraram-se como telófase I os oócitos que apresentaram fuso celular alongado, perpendicular à membrana oocitária, com cromossomos distribuídos nas suas extremidades. Foi considerada ativação partenogenética a presença de extrusão do segundo corpúsculo polar.

Se os cromossomos apresentavam-se dispersos, desalinhados ou fragmentados, se a cromatina feminina encontrava-se aglomerada com microtúbulos interfásicos, se o formato do fuso não estava normal (forma de barril) ou se o mesmo apresentava ruptura, este foi considerado anormal.

A comparação entre as taxas de MIV e a proporção de anormalidades do fuso celular e distribuição cromossômica entre os dois grupos estudados foi realizada pelo teste exato de Fisher. O grau de significância (alfa) foi definido em 0,05 (bicaudal), sendo que o valor de $\mathrm{p}<0,05$ foi considerado estatisticamente significativo.

\section{Resultados}

No grupo de estudo, dos 21 oócitos imaturos submetidos à MIV, dez atingiram a MII, caracterizada pela extrusão do primeiro corpúsculo polar. No Grupo Controle, 13 dos 29 oócitos imaturos submetidos a MIV atingiram a MII. As taxas de MIV foram similares entre os dois grupos avaliados ( 47,6 e $44,8 \%$, respectivamente, nos Grupos SOP e Controle).

Por dificuldades técnicas durante a execução do protocolo de imunofluorescência, um oócito do Grupo Controle não pôde ser analisado. Desta forma, foram analisados dez oócitos do grupo de estudo (SOP) e 12 oócitos do Grupo Controle, que, segundo análise por microscopia óptica, estavam em MII. No grupo de estudo, dos dez oócitos avaliados, foram observados três oócitos normais em MII (30\%), seis MII anormais (60\%) e um em estágio de telófase I normal (10\%). No Grupo Controle, dos 12 oócitos analisados, foram observados quatro MII normais $(33,3 \%)$, quatro MII anormais $(33,3 \%)$, um oócito em telófase I normal $(8,4 \%)$ e três sofreram ativação partenogenética (25\%). Não foi detectada diferença significativa na incidência de anomalias meióticas entre os dois grupos avaliados (Tabela 1).

\section{Discussão}

O presente estudo foi o primeiro na literatura que avaliou as proporções de anomalias meióticas nos oócitos

Tabela 1 - Resultados da análise do fuso celular e configuração cromossômica de oócitos maturados in vitro, obtidos de ciclos estimulados de pacientes com síndrome dos ovários policísticos (SOP) e infertilidade por fatores masculino e/ou tubário (Grupo Controle)

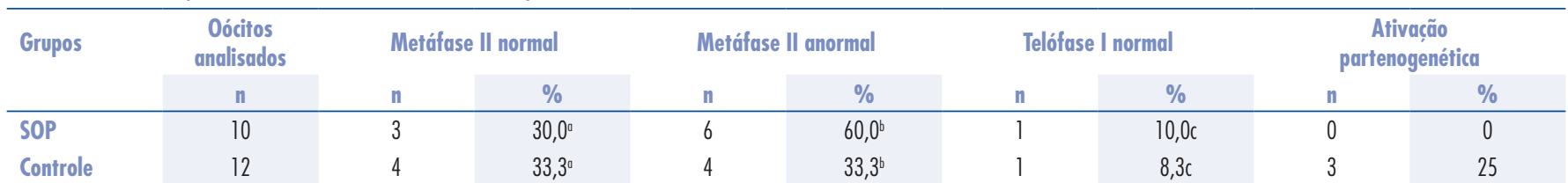

Oócitos analisados: oócitos imaturos, obtidos em ciclos estimulados com gonadotrofinas, que, após maturação in vitro, apresentaram a extrusão do primeiro corpúsculo polar; $n=$ número.

Mesmos sobrescritos ${ }^{a, b, c}$ dentro da mesma coluna indicam ausência de diferença significativa ( $p>0,05$; teste exato de Fisher). 
maturados in vitro provenientes de ciclos estimulados de pacientes com SOP, comparando-as aos de mulheres com fatores tubário e/ou feminino de infertilidade. Os dados do presente estudo não evidenciaram diferença significativa nas frequiências de anomalias meióticas entre estes dois grupos estudados. Contudo, sugerem tendência a maior ocorrência de anomalias meióticas nos oócitos de mulheres com SOP. Se confirmados, os referidos achados poderão contribuir para a elucidação de um dos mecanismos envolvidos na sugerida piora da qualidade oocitária em portadoras desta afecção, quando submetidas à estimulação ovariana para a realização de TRA de alta complexidade.

$\mathrm{O}$ uso de oócitos humanos maduros em pesquisas utilizando metodologias invasivas, como a microscopia de imunofluorescência, que requer a fixação e coloração do material, inviabilizando-o para o uso clínico, depararse-ia com a grande dificuldade de doação e a conseqüente escassez de material. Por isso, optou-se pela utilização de oócitos imaturos, obtidos após captação ovariana em ciclos estimulados com gonadotrofinas endógenas, que, após período pré-definido de MIV, apresentaram a extrusão do primeiro corpúsculo polar. Todavia, a baixa proporção de oócitos imaturos obtidos nos ciclos estimulados (16,7 e 12,4\% de oócitos imaturos, respectivamente, nos Grupos Controle e SOP), associada à necessidade de que apresentassem a extrusão do primeiro corpúsculo polar após MIV, para que fossem utilizados no presente estudo, justificou a pequena casuística obtida após um ano de inclusão de pacientes elegíveis para participarem do presente estudo.

Não se observou diferença significativa nas taxas de MIV entre os dois grupos avaliados. Utilizando meios e condições de cultivo previamente descritos, obtiveramse taxas de MIV de 47,6 e 44,8\%, respectivamente, nos Grupos SOP e Controle, abrindo perspectivas de obtenção de cerca de $50 \%$ de oócitos maduros a partir da realização de MIV de oócitos imaturos, obtidos em ciclos estimulados. Esta estratégia poderia ter utilidade prática, sobretudo nos casos em que forem captados poucos oócitos maduros e forem dispostos oócitos imaturos, que, habitualmente, não são utilizados na prática clínica, possibilitando a obtenção de um maior número de embriões passíveis de serem transferidos a fresco e/ou criopreservados. Estudos futuros serão importantes para analisar a viabilidade e a competência destes oócitos maturados in vitro, obtidos em ciclos estimulados, para suportar o desenvolvimento embrionário subseqüente.

Todavia, é importante ressaltar-se que, apesar de a visualização da extrusão do primeiro corpúsculo polar ter sido o critério morfológico utilizado no presente estudo para caracterizar a maturidade oocitária, o que é largamente empregado nas clínicas de reprodução assistida, observou-se a presença de oócitos em telófase I (oócitos que não completaram a meiose I) em ambos os grupos avaliados (10 e 8,4\%, respectivamente, nos Grupos SOP e Controle). Ressaltou-se que as taxas de fertilização de oócitos em telófase I parecem ser inferiores às observadas após a inseminação de oócitos em MII e que a utilização da análise não invasiva do fuso meiótico oocitário, por meio da microscopia de polarização, poderia contribuir para a identificação dos oócitos que, apesar de apresentarem a extrusão do primeiro corpúsculo polar, ainda não completaram a meiose $\mathrm{I}^{19,20}$.

Ressaltou-se que os nossos dados evidenciaram um elevado percentual de anomalias meióticas, caracterizadas por anormalidades do fuso e/ou distribuição cromossômica oocitárias, tanto nos oócitos em MII do Grupo Controle $(33,3 \%)$ como no grupo de estudo $(60 \%)$. Não se dispuseram de dados na literatura avaliando a incidência de anomalias meióticas em oócitos humanos, maturados in vitro, obtidos de ciclos estimulados com gonadotrofinas. Alguns autores, realizando análise citogenética de oócitos em MII, não fertilizados após a realização de fertilização in vitro convencional, detectaram anomalias cromossômicas em 24,6\% dos oócitos de portadoras de SOP ${ }^{21}$. Porém, tanto o material como as metodologias empregadas foram distintas das utilizadas no presente estudo, impossibilitando a adequada comparação dos dados obtidos. Encontrou-se um único estudo publicado avaliando a incidência de anomalias do fuso meiótico e configuração cromossômica em oócitos humanos maturados in vitro, obtidos em ciclos não estimulados com gonadotrofinas, evidenciando, respectivamente, 43,7 e 33,3\% de anormalidades ${ }^{22}$. Todavia, os oócitos maturados in vitro analisados foram obtidos em ciclos não estimulados com gonadotrofinas. Como se postula um potencial papel deletério da estimulação ovariana com gonadotrofinas na dinâmica do citoesqueleto e competência oocitária ${ }^{23}$, é possível que a incidência de anomalias meióticas seja maior nos oócitos maturados in vitro, obtidos de ciclos estimulados, do que naqueles não estimulados, o que precisa ser mais bem avaliado por meio de estudos utilizando metodologia pertinente. Este mesmo estudo ${ }^{22}$, comparando a incidência de anormalidades meióticas entre os oócitos maturados in vitro, oriundos de ciclos não estimulados, com os maturados in vivo, obtidos em ciclos estimulados, encontrou uma proporção significativamente maior de anomalias meióticas nos oócitos maturados in vitro, sugerindo que a MIV tenha um efeito deletério sobre a organização do fuso celular e o alinhamento cromossômico oocitário. Desta forma, é provável que a incidência de anomalias meióticas obtidas no presente estudo (60\%), no qual se analisaram oócitos maturados in vitro, obtidos de ciclos estimulados com gonadotrofinas, seja superior tanto à observada nos oócitos maturados in vivo, utilizados rotineiramente nas TRA, como à evidenciada em oócitos maturados in vitro, porém provenientes de ciclos não 
estimulados $^{22}$. Todavia, como se compararam oócitos de pacientes com SOP com os de pacientes com fatores tubário e/ou masculino de infertilidade (Grupo Controle), ambos maturados in vitro, e encontrou-se uma tendência a maior incidência de anomalias meióticas nas portadoras de SOP, estes achados sugerem um possível comprometimento da qualidade oocitária ralacionada à SOP, e não apenas à MIV, o que deverá ser mais bem analisado, aumentando-se a presente casuística.

Os dados preliminares do presente estudo não evidenciaram diferença significativa nas taxas de MIV e nas freqüências de anomalias meióticas entre os oócitos maturados in vitro provenientes de ciclos estimulados de pacientes com SOP quando comparados aos de mulheres com fatores tubário e/ou masculino de infertilidade. Contudo, sugerem uma tendência a maior ocorrência de anomalias meióticas, caracterizadas por anormalidades do fuso e/ou distribuição cromossômica oocitárias, nos oócitos de pacientes com SOP, o que poderá ser elucidado ampliando-se a presente casuística. Se forem confirmados, estes achados podem apontar um possível papel destas anormalidades, relacionadas à maturação nuclear oocitária, nas controversas menores taxas de fertilização observadas em pacientes com SOP submetidas às TRA. Se não confirmados, sugerirão o possível envolvimento de fatores relacionados à maturação citoplasmática, e não nuclear, na etiopatogênese da piora da qualidade oocitária em portadoras de SOP.

\section{Agradecimentos}

Agradecemos a contribuição das funcionárias do laboratório de reprodução assistida: Maria Aparecida Carneiro Vasconcelos, Marilda Yamada Dantas, Roberta Cristina Giorgenon, Maria Auxiliadora Pádua Rosa e Sandra Viana, pelo apoio técnico ao trabalho.

\section{Referências}

1. Heijnen EM, Eijkemans M, Hughes EG, Laven JS, Macklon NS, Fauser BC. A meta-analysis of outcomes of conventional IVF in women with polycystic ovary syndrome. Hum Reprod Update. 2006;12(1):13-21.

2. Homburg R. Pregnancy complications in PCOS. Best Pract Res Clin Endocrinol Metab. 2006;20(2):281-92.

3. Giudice LC. Endometrium in PCOS: implantation and predisposition to endocrine CA. Best Pract Res Clin Endocrinol Metab. 2006;20(2):235-44.

4. Patel SS, Carr BR. Oocyte quality in adult polycystic ovary syndrome. Semin Reprod Med. 2008;26(2): 196-203.

5. Regan L, Owen EJ, Jacobs HS. Hypersecretion of luteinising hormone, infertility, and miscarriage. Lancet. 1990;336(8724):1141-4.

6. Sahu B, Ozturk O, Ranierri M, Serhal P. Comparison of oocyte quality and intracytoplasmic sperm injection outcome in women with isolated polycystic ovaries or polycystic ovarian syndrome. Arch Gynecol Obstet. 2008;277(3):239-44.

7. Ludwig M, Finas DF, al-Hasani S, Diedrich K, Ortmann O. Oocyte quality and treatment outcome in intracytoplasmic sperm injection cycles of polycystic ovarian syndrome patients. Hum Reprod. $1999 ; 14(2): 354-8$.

8. Aboulghar MA, Mansour RT, Serour GI, Ramzy AM, Amin YM. Oocyte quality in patients with severe ovarian hyperstimulation syndrome. Fertil Steril. 1997;68(6):1017-21.

9. Kodama H, Fukuda J, Karube H, Matsui T, Shimizu Y, Tanaka T. High incidence of embryo transfer cancellations in patients with polycystic ovarian syndrome. Hum Reprod. 1995; 10(8): 1962-7.

10. Tarlatzis BC, Grimbizis G, Pournaropoulos F, Bontis J, Lagos S, Spanos $E$, et al. The prognostic value of basal luteinizing hormone: follicle-stimulating hormone ratio in the treatment of patients with polycystic ovarian syndrome by assisted reproduction techniques. Hum Reprod. 1995; 10(10):2545-9.
11. Wang WH, Meng L, Hackett RJ, Keefe DL. Developmental ability of human oocytes with or without birefringent spindles imaged by Polscope before insemination. Hum Reprod. $2001 ; 16(7): 1464-8$

12. Moon JH, Hyun CS, Lee SW, Son WY, Yoon SH, Lim JH. Visualization of the metaphase II meiotic spindle in living human oocytes using the Polscope enables the prediction of embryonic developmental competence after ICSI. Hum Reprod. 2003; 18(4):817-20.

13. Shen Y, Betzendahl I, Tinneberg HR, Eichenlaub-Ritter U. Enhanced polarizing microscopy as a new tool in aneuploidy research in oocytes. Mutat Res. 2008;651 (1-2):131-40.

14. Navarro PA, Liu L, Trimarchi JR, Ferriani RA, Keefe DL. Noninvasive imaging of spindle dynamics during mammalian oocyte activation. Fertil Steril. 2005;83 Suppl 1:1197-205.

15. Miyara F, Aubriot FX, Glissant A, Nathan C, Douard S, Stanovici $A$, et al. Multiparameter analysis of human oocytes at Metaphase II stage after IVF failure in non-male infertility. Hum Reprod. 2003; 18(7): 1494-503.

16. The Rotterdam ESHRE/ASRM-Sponsored PCOS Consensus Workshop Group. Revised 2003 consensus on diagnostic criteria and long-term health risks related to polycystic ovary syndrome. Fertil Steril. 2004;81(1):19-25.

17. Allworth AE, Albertini DF. Meiotic maturation in cultured bovine oocytes is accompanied by remodeling of the cumulus cell cytoskeleton. Dev Biol. 1993;158(1):101-12.

18. Liu L, Oldenbourg R, Trimarchi JR, Keefe DL. A reliable, noninvasive technique for spindle imaging and enucleation of mammalian oocytes. Nat Biotechnol. 2000; 18(2):223-5.

19. Hyun CS, Cha JH, Son WY, Yoon SH, Kim KA, Lim JH. Optimal ICSI timing after the first polar body extrusion in in vitro matured human oocytes. Hum Reprod. 2007;22(7):1991-5. 
20. Madaschi C, Carvalho de Souza Bonetti T, Paes de Almeida Ferreira Braga D, Pasqualotto FF, laconelli A Jr, Borges E Jr. Spindle imaging: a marker for embryo development and implantation. Fertil Steril. In press 2007.

21. Sengoku K, Tamate K, Takuma N, Yoshida T, Goishi K, Ishikawa M. The chromosomal normality of unfertilized oocytes from patients with polycystic ovarian syndrome. Hum Reprod. 1997; 12(3):474-7.
22. Li Y, Feng HL, Cao YJ, Zheng GJ, Yang Y, Mullen S, et al. Confocal microscopic analysis of the spindle and chromosome configurations of human oocytes matured in vitro. Fertil Steril. 2006;85(4):827-32.

23. Lee ST, Han HJ, Oh SJ, Lee EJ, Han JY, Lim JM. Influence of ovarian hyperstimulation and ovulation induction on the cytoskeletal dynamics and developmental competence of oocytes. Mol Reprod Dev. 2006;73(8): 1022-33. 